\title{
Probióticos: desafíos, revisión y alcance
}

\section{Probiotics: challenges, review and scope}

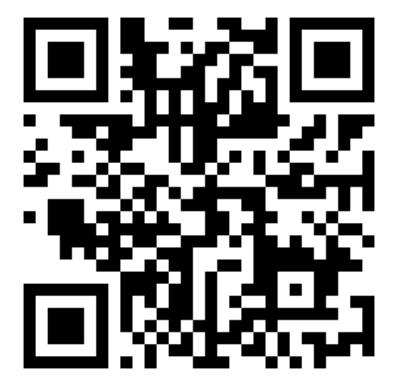

Recibido $10 / 05 / 2021$

\author{
${ }^{1}$ Dra. Renata Rappaccioli Salinas \\ Investigadora independiente, San José, Costa Rica \\ (iD) https://orcid.org/0000-0002-2646-8387
}

${ }^{2}$ Dra. Verónica Zaror Loaiciga Investigadora independiente, San José, Costa Rica

(iD) https://orcid.org/0000-0003-3594-1954

${ }^{3}$ Dr. Santiago Herrera Jaramillo Investigador independiente, San José, Costa Rica (iD) https://orcid.org/0000-0003-1562-0248

$\begin{array}{lr}\text { Corregido } & \text { Aceptado } \\ 17 / 05 / 2021 & 20 / 05 / 2021\end{array}$

\section{RESUMEN}

Los probióticos son microorganismos vivos no patógenos con efectos beneficiosos para la salud del huésped. Las especies y cepas más usadas pueden ser parte de la composición de distintos tipos de productos, entre los que se incluyen alimentos, medicamentos y suplementos alimenticios que sobreviven en el tránsito del tubo gastrointestinal. Participan en la prevención y tratamiento de enfermedades infecciosas digestivas, hepáticas, afecciones autoinmunes, homeostasis intestinal, enfermedades óseas entre otras.

PALABRAS CLAVE: microorganismos; probióticos; microbiota; suplementos alimenticios.

\section{ABSTRACT}

Probiotics are non-pathogenic live microorganisms with beneficial effects on the health of the host. The most widely used species and strains can be part of the composition of different types of products, including food, drugs and dietary supplement that survive in the transit of the gastrointestinal tract. They participate in the prevention and treatment of infectious digestive and liver diseases, autoimmune conditions, intestinal homeostasis, bone diseases, among others.

KEYWORDS: microorganisms; probiotics; microbiota; dietary supplement.

${ }^{1}$ Médica general, graduada de la Universidad de Centro América (UACA). Cód. MED16958. Correo: renata.rappaccioli@hotmail.com

${ }^{2}$ Médica general, graduada de la Universidad de Centro América (UACA). Cód. MED16302.

${ }^{3}$ Médico general, graduado de la Universidad de Centro América (UACA). Cód. MED16959. 


\section{INTRODUCCIÓN}

Se puede definir a los probióticos como un grupo de medicamentos conformado por microorganismos vivos, los cuales, administrados en cantidades adecuadas, producen beneficios para el paciente. La mayoría de probióticos son aislados de la microbiota del ser humano, y pueden administrarse para aumentar o reforzar la microbiota humana, existen múltiples géneros de probióticos que provienen de microorganismos bacterianos como fúngicos. Para poder definirse como probiótico, un medicamento debe de cumplir con ciertas características, por ejemplo, deben poseer la capacidad de atravesar el tracto gastrointestinal hasta el área colónica donde realizan su mecanismo de acción. Además, deben de poder ser identificables en cuanto a su género y especie $(1,2)$.

A pesar de no contar con muchos estudios clínicos in vivo en humanos, se ha demostrado que los probióticos efectúan su mecanismo de acción por diferentes vías, por ejemplo, disminuyendo el pH intraluminal, actuando como barrera intestinal al ocasionar inhibición competitiva, incrementando la producción de inmunoglobulinas $A$ y activando la proteína cinasa $\mathrm{C}$ para fortalecer los sitios de unión $(1,2)$.

Es importante destacar que la FDA (Food and Drug Administration) no ha aprobado el uso de ningún probiótico como terapéutico. De ahí nace el objetivo de este artículo el cual busca explorar los tipos de probióticos disponibles en la actualidad, su eficacia tanto dentro como fuera del tracto gastrointestinal $(1,2)$.

\section{MÉTODO}

Se realiza una investigación a través de las bases de datos Scielo, UpToDate, Medline y el grupo Crochrane database sobre las más recientes actualizaciones en el tema de probióticos y su relevancia como tratamiento para distintas patologías. Así como también se documenta sobre las nuevas disposiciones de la AGA en cuanto al uso de probióticos. Se revisaron artículos, revistas y estudios redactados en el idioma inglés y español.

\section{¿CÓMO ACTÚAN LOS PROBIÓTICOS?}

Un verdadero probiótico debe ser preferiblemente de origen humano, seguro, libre de vectores que logren transferir resistencia a los antibióticos y de factores de toxicidad. Además, deben tener la capacidad de sobrevivir en condiciones intestinales $(\mathrm{pH}$, enzimas, ácidos, sales biliares, etc.), estimular el sistema inmune y de mostrar antagonismo contra patógenos (3).

La formación de la flora intestinal comienza antes del parto y continúa hasta la infancia. Varios factores influyen en la colonización intestinal inicial, tales como la genética, método de parto, uso de antibiótico, alimentación, procesos inflamatorios, bacterias de la placenta, meconio y leche materna. Después de la administración y consumo de las cepas de probióticos, empieza el proceso de colonización $(3,4)$.

Para una adecuada colonización, se requiere que la cepa microbiana tenga adhesión a la mucosa intestinal y así lograr una mayor interacción con el huésped. Las células epiteliales del intestino secretan mucina para evitar la adhesión de las bacterias patógenas, varias proteínas del lactobacillus promueven esta adhesión $(3,4)$. En adultos sanos, el uso de probióticos aumenta la producción de AGCC (ácidos grasos de cadena corta). Estos producen efectos biológicos potenciales en humanos como por ejemplo (3)(4): 
- Cambios en la excitabilidad neuronal aumentando la saciedad.

- Aumento en la sensibilidad de la insulina y disminución del acúmulo de lípidos a nivel de hígado y muscular.

- Disminución de la lipolisis.

- Regulación de homeostasis y metabolismo de la energía.

Otro de los principales mecanismos de acción de los probióticos es contribuir a la regulación de la respuesta inmune del huésped, modificando actividades enzimáticas relacionadas con la metabolización de varios carcinógenos y suprimiendo la inflamación crónica. La flora intestinal modula el sistema inmunológico a través de producción de moléculas que son capaces de estimular las células inmunes. Estos efectos inmunomoduladores se deben a la interacción de bacterias probióticas y células epiteliales con monocitos, macrófagos y linfocitos, induciendo así el desarrollo de células t-reguladoras (4-6).

\section{TIPOS DE PROBIÓTICOS}

Al hablar de la clasificación de los probióticos se debe tener en cuenta que se pueden dividir tanto por etiología (bacterias y hongos) como en composición (un solo microorganismo o varios). La familia de bacterias más utilizada para este fin es la familia Lactobacillus, dentro de los cuales cabe destacar $L$. reuteri, $L$. casei, $L$. rhamnosus GG, L.bulgaricus y $L$. acidophillus. Al hablar del otro tipo de probióticos, los más importantes son las levaduras de Saccharinyces boulardii. Otros grupos de bacterias utilizados son Bifidobacterium y Streptococcus termophillus. También existen otros productos que se catalogan como probióticos, por ejemplo, los yogures que contienen microorganismos productores de lactasa, con lo cual se cree que mejoran la tolerancia a la lactosa $(7,8)$.
Existe sin embargo dificultad a la hora de clasificar a los probióticos, debido a múltiples factores, por ejemplo, a nivel comercial se encuentran inconsistencias en los nombres de microorganismos a nivel taxonómico, no existe una estricta supervisión sobre el proceso de formulación de los probióticos, la vida útil o el número de probióticos disponibles en cada vial. También existe el inconveniente de la gran cantidad de productos que se comercializan bajo el nombre de probióticos sin estudios científicos que los avalen como tal $(7,8)$.

En los Estados Unidos, el ente regulador FDA a pesar de no certificar el uso de probióticos a nivel terapéutico, puede brindar la calificación de GRAS (generalmente se reconoce como seguro). Dentro de los probióticos con esta clasificación se encuentran por ejemplo $(7,8)$ :

- L. acidophilus

- Lactobacillus lactis

- Pediococcus acidilactici

- L. rhamnosus cepa GG

- L. casei cepa Shirota

- Lactobacillus fermentum cepa CECT5716

- Lactobacillus plantarum cepa 299v

- L. reuteri cepa DSM 17938

- $\quad$ L. reuteri cepa NCIMB 30242

- L. rhamnosus cepa HN001

- L. rhamnosus cepa HN001 producido en un medio con leche

\section{BENEFICIOS}

Los beneficios de los probióticos se pueden dividir en inmunológicos y no inmunológicos, entre los que modulan la respuesta inmune del paciente tendríamos activación de los macrófagos locales y las células dendríticas para aumentar la presentación de antígenos a los linfocitos B, desencadenando la producción de IgA secretora tanto local 
como sistémica, pueden también modular los perfiles de las citoquinas para desencadenar una respuesta tolerogénica ante la flora comensal o prebiótica o proinflamatoria ante la presencia de patógenos (9).

Entre los beneficios no inmunológicos se incluye que digieren los alimentos $y$ compiten con los patógenos por los alimentos, alteran el $\mathrm{pH}$ local para crear un ambiente desfavorable para los patógenos, producen sustancias bactericidas $y$ bacteriostáticas que eliminan o inhiben a los patógenos, estimulan la producción epitelial de moco, compiten por los sitios de adhesión al moco y al epitelio intestinal, aumentan la función de barrera intestinal mediante efectos antioxidantes $(9,10)$.

Los probióticos además contribuyen a patologías como intolerancia a la lactosa ya que los lactobacilos la degradan e impiden así que llegue sin digerir al intestino grueso y que inicie con los síntomas asociados a mala digestión como los son flatulencia, distensión abdominal y diarrea $(9,10)$.

Se ha evidenciado una reposición de la microbiota después que la que era residente o autóctona haya sido eliminada ya sea por diarrea asociada al tratamiento con antibióticos o diarreas en niños producidas por virus. Por lo que según este principio también se han observado beneficios como prevención de recidivas de vaginosis y de vaginitis al administrar lactobacilos probióticos posterior a un tratamiento antibiótico (9).

\section{CONSUMO}

La principal vía de administración de los microorganismos probióticos es la vía oral, los cuales deben sobrevivir a lo largo del tracto gastrointestinal, tolerar el ácido, la bilis y las enzimas a nivel gástrico y lograr posteriormente adherirse al epitelio intestinal, además deben garantizar la supervivencia de los microorganismos en forma vial durante el almacenamiento hasta el final de la vida útil $(3,11)$.

Para obtener los beneficios deseados de los probióticos hay que tener en cuenta la especificidad de la cepa o la localización de la enfermedad diana y más cuidadosamente la dosis, ruta y duración de la frecuencia de la administración. Lo recomendable es que el suplemento tenga entre 2 y 10 billones unidades de colonias formadoras (UFC), dependiendo de la presentación de cada envase y cepa, siendo importante escoger el mejor tipo de bacteria que posee para el tipo de problema a tratar $(11,12)$.

Se pueden obtener probióticos a través de diferentes maneras, ya sea consumiendo alimentos considerados como probióticos naturales los cuales han pasado por un proceso de "lactofermentación", por ejemplo, el yogurt o el kéfir y la otra manera es a través de suplementos con probióticos. Cuanto mayor es la diversidad de probióticos, así como el número de bacterias dentro de cada comprimido, mejor será el suplemento y más rápido será el beneficio deseado $(3,11)$.

Es importante probar el suplemento durante unas cuatro semanas, si no se observa mejoría lo ideal sería probar una cepa diferente. Deben ser ingeridos antes 0 después de una comida, para que los alimentos sean de ayuda para las bacterias a sobrevivir por el paso del ácido gástrico y así llegar hasta el intestino. También se aconseja el consumo de alimentos ricos en fibras ya que son el principal alimento para los probióticos, favoreciendo su supervivencia en el intestino (12).

\section{PREVENCIÓN Y TRATAMIENTO DE LA ENFERMEDAD DIARREICA AGUDA}

Existen múltiples estudios del efecto de los probióticos sobre la enfermedad diarreica aguda, la gran mayoría en población pediátrica. Tales como la AAP (Asociación 
Americana de Pediatría) y la AGA (Asociación Americana de Gastroenterología) que cuentan con guías para el correcto uso de probióticos para tratar la enfermedad diarreica aguda dependiendo de la etiología de la misma. $(2,13)$.

En una revisión técnica de la AGA acerca del rol de los probióticos en el manejo de desórdenes gastrointestinales se estudió el papel que pueden tener los probióticos en prevenir la diarrea asociada a la infección por Clostridium. difficile (CDAD) en pacientes recibiendo antibioticoterapia oral o IV donde se determinó que los probióticos con múltiples cepas de microorganismos tienen una mayor probabilidad de prevenir la $C D A D$, mientras que los que tenían una sola cepa, aquellos conformados por la levadura de $S$. boulardii, fueron los únicos que demostraron disminuir la incidencia de $\operatorname{CDAD}(2,13)$.

En otra revisión realizada por Cochrane sobre la eficacia de los probióticos en enfermedades infecciosas, donde se pudo determinar que las cepas más efectivas en acortar la duración de la diarrea infecciosa y en reducir la frecuencia de las deposiciones fueron L. casei cepa GG, S Boulardii, Bacterias productoras de ácido láctico (BAL) $y$ enterococos SF68. Sin embargo, se menciona que se necesitan más estudios específicos según grupo etario y probiótico seleccionado para llegar a brindar mejores recomendaciones $(2,13)$.

El impacto de los probióticos en pacientes con gastroenteritis aguda (EDA) se puede definir como incierto, esto debido a la amplia cantidad de estudios realizados con una gran variedad de probióticos, además según la revisión técnica de AGA, en cuanto al rol de los probióticos en el manejo de desórdenes gastrointestinales, se debe tomar en cuenta el rol fundamental de la etiología de la gastroenteritis aguda, la cual varía según región geográfica, la mayoría de estudios han sido realizados en Europa y
Asia, dicha revisión técnica no encontró estudios en EEUU o en Canada que reflejaran beneficios en el uso de probióticos en pacientes con EDA $(2,13)$.

\section{PROBIÓTICOS EN ENFERMEDAD INFLAMATORIA INTESTINAL}

La AGA realizó una publicación sobre las recomendaciones oficiales del uso de probióticos en patologías gastrointestinales desarrollado por AGA Institute Clinical Guidelines Committee y aprobado por AGA Governing Board en agosto 2020, entre las patologías gastrointestinales que se mencionan se encuentra la Enfermedad Inflamatoria Intestinal $(14,15)$.

En adultos y niños con la enfermedad de Crohn y colitis ulcerativa se recomienda el uso de probióticos solo en contexto de ensayo clínico. Se realizaron estudios utilizando cepas como Escherichia coli, $S$ boulardii, Lactobacillus johnsonii, B. breve Yakult, B. bifidum Yakult entre otras. Los resultados no mostraron evidencia de ningún beneficio con el uso de probióticos para la remisión de la enfermedad, por lo que se sugiere continuar la investigación para identificar poblaciones específicas $(14,15)$.

\section{RITMO INTESTINAL}

Las manifestaciones más frecuentes de los cambios en el ritmo intestinal son la diarrea y el estreñimiento. Los trastornos en el ritmo intestinal tienen una elevada prevalencia en la sociedad actual y se presentan de maneras diferentes, dependiendo de hábitos dietéticos, factores ambientales, estilo de vida, flora bacteriana, etc. (16).

Entre los probióticos que han demostrado efectos beneficiosos para el tránsito intestinal y mejorando los síntomas de constipación destaca el Bifidobacterium lactis, el cual promueve el desarrollo de una microbiota intestinal saludable mediante el 
incremento de la cantidad total de bifidobacterias (16).

Abundante Flora intestinal de bifidobacterias y lactobacilos realiza efectos positivos sobre la función inmune y barrera. Estimulan la maduración y la funcionalidad de las células inmunes a través de sus metabolitos, influyendo así en la motilidad intestinal y causando beneficio en individuos con estreñimiento (16).

\section{PROBIÓTICOS EN PACIENTES UCI Y QUIRÚRGICOS}

Se han realizado diferentes estudios aleatorizados en pacientes sometidos a cirugía mayor abdominal (incluyendo politraumatizados, cáncer, cirugía de estómago, colon etc.), comparando generalmente simbióticos adicionados a la nutrición enteral frente a la fibra probiótica contenida en los preparados y frente a otro grupo control con nutrición parenteral o enteral (estándar con fibra, peptídica o con glutamina). En algunos estudios, pero no en todos, los preparados simbióticos redujeron la incidencia de infecciones bacterianas respecto a la nutrición parenteral total o respecto a otras formulaciones de nutrición enteral $y$ con resultados intermedios respecto a la fibra prebiótica $(17,18)$.

Las guías canadienses de práctica clínica sobre nutrición en pacientes críticos recomiendan valorar el empleo de probióticos en pacientes de $\mathrm{UCl}$ en base a una reducción de las infecciones y una tendencia a disminución de neumonías asociada a ventilación mecánica y de la estancia en $\mathrm{UCl}$, sin efectos sobre la mortalidad. Sí resaltan que no se debería usar S. boulardii en pacientes críticos, por el riesgo de infección fúngica $(17,18)$.

En un metaanálisis realizado en pacientes de UCl se ha evaluado el uso de combinación de Bifidobacterium longum $+\mathrm{L}$. bulgaricus $+\mathrm{S}$. thermophilus vs un grupo control (glutamina + fibra fermentable) en la prevención de la neumonía asociada a ventilación mecánica, objetivándose un descenso significativo en su incidencia. No obstante, de nuevo, dado que la calidad de la evidencia es baja y dada la alta heterogeneidad en los resultados, no pueden extraerse conclusiones definitivas. $(17,18)$.

Sin embargo, en el estudio PROPATRIA, de 2009, que empleó el probiótico Ecologic 641 compuesto de seis cepas de bacterias: $L$. acidophilus, L. casei, Lactobacillus salivarius, $L$. lactis y dos bifidobacterias (Bifidobacterium bifidum y $B$. lactis) en una dosis total de 1010 bacterias vivas administrado junto con una fórmula enteral con mezcla de fibras, se observó un aumento significativo de la mortalidad en el grupo de probióticos (16\% frente 6\%). En el subgrupo de sujetos con pancreatitis necrosante falleció el $41 \%$ en el grupo de probióticos, frente al $15 \%$ en el grupo placebo. Nueve pacientes sufrieron isquemia mesentérica (ocho fallecieron) en el grupo de probióticos $(17,18)$.

\section{SALUD ÓSEA}

La flora bacteriana o la microbiota ha desempeñado un papel importante en la regulación de numerosas funciones fisiológicas. $Y$ el hueso es un tejido que no escapa de esta relación, confirmándose el impacto de la microbiota en su regulación metabólica (19).

Los probióticos de los géneros lactobacillus y bifidobacterium han sido los más conocidos en ámbitos de la salud ósea. Se ha comprobado que estas bacterias tienen la capacidad de estar envueltas en la producción de vitaminas, D, C y K, todas ellas importantes para el mantenimiento de la densidad ósea. Otro mecanismo es la influencia de los SCFA (ácidos grasos de cadena corta) contribuyendo a la disminución de la resorción ósea (19). 
Por otro lado, la disminución de esta causa un aumento de permeabilidad intestinal por debilitación de la barrera. Esta circunstancia lleva a un aumento de translocación bacteriana y de células proinflamatorias, lo cual es parte del mecanismo responsable de la resorción ósea que se observa cuando se reducen los niveles estrogénicos (19).

Por último, se ha evidenciado que el Lactobacillus helveticus aumenta la reabsorción intestinal de calcio, causando un aumento en la masa ósea. Siendo beneficiosos en múltiples enfermedades reumatológicas tales como, artritis reumatoide, artritis crónica juvenil, artritis psoriásica o espondiloartritis (19).

\section{EFECTOS DE LOS PROBIÓTICOS EN EL SÍNDROME METABÓLICO}

Para hablar del efecto de los probióticos en el síndrome metabólico, se debe primero definir este síndrome, según la Organización Mundial de la Salud este se define como la presencia de alguno de 2 de los siguientes: intolerancia a la glucosa, diabetes tipo 2 e insulinoresistencia y por lo menos 2 de los siguientes: alteración antropométrica (circunferencia abdominal $\geq 90 \mathrm{~cm}$ hombres, 85 mujeres ó un IMC $\geq 30$ ), alteración lipídica (TG $\geq 150 \mathrm{mg} / \mathrm{dl}$, HDLc $<35 \mathrm{mg} / \mathrm{dl}$ hombres ó < $39 \mathrm{mg} / \mathrm{dl}$ mujeres), presión arterial Sistólica $\geq 140 \mathrm{mmhg}$ o diastólica $\geq$ $90 \mathrm{mmhg}$, microalbuminuria $\geq 20 \mu \mathrm{g} / \mathrm{min}$ ó relación albumina/creatinina $>30 \mathrm{mg} / \mathrm{g}$ $(20,21)$.

Juegan un rol fundamental la cantidad y calidad de alimentos que se ingieren, los cuales al atravesar el tracto gastrointestinal afectan a los organismos que van a predominar y estos con sus metabolitos a su vez, influyen en la aparición de diferentes enfermedades. Existen múltiples factores que han alterado nuestra microbiota en la actualidad, entre los cuales destacan: un mayor consumo de grasas saturadas, de azúcares refinados, el incorrecto uso de antibioticoterapia. Estos cambios llevan indudablemente a la reducción en la diversidad de las microbiota intestinal, de ahí que se ha visto que rescatar y regresar esta microbiota no solo puede alterar el fenotipo del obeso y las consecuencias metabólicas que consigo trae, sino que también tiene un efecto sobre la expresión de ciertos genes como tejido adiposo, hígado, musculo ó intestino $(20,21)$.

En un estudio realizado en Argentina donde se analizó a estudiantes con una edad promedio de 24 años se demostró que el grupo de estudiantes que consumía probióticos tuvo niveles menores de colesterol total, glucemia y HDLc, así como riesgo o presencia de síndrome metabólico. $(20,21)$.

Se puede definir que el consumir probióticos resulta en la reducción del colesterol en la sangre lo cual repercute de manera positiva en la disminución de eventos cerebro y cardiovasculares, así como en la prevención de la obesidad y la diabetes, debido a esto se puede deducir que el impacto del consumo de probióticos a nivel bioquímico confiere protección contra el síndrome metabólico $(20,21)$.

\section{CONCLUSIONES}

- Las investigaciones realizadas a nivel mundial por expertos en todas las áreas del sector salud, han arrojado innumerables beneficios de los probióticos en salud ósea, ritmo intestinal, EDA, pacientes quirúrgicos, síndrome metabólico, basadas en el antagonismo antimicrobiano, restauración del balance de la microbiota y mejoría a la respuesta inmune.

- Debido a que el nivel de evidencia se calificó como bajo en los estudios realizados en pacientes con Ell, se concluyó que se requieren de mayor cantidad de estudios para definir poblaciones específicas con Ell que se 
verían beneficiadas con probióticos, y así también las cepas más efectivas para cada caso.

- En cuanto al efecto de los Probióticos sobre la gastroenteritis aguda según la mayoría de los estudios analizados en este artículo, no se encontró diferencia significativa entre los grupos administrados placebos vs los administrados probióticos. Sin embargo, cabe destacar que en una revisión Cochrane, se encontró que las únicas cepas en disminuir tanto frecuencia como duración de la diarrea infecciosa fueron: Lactobacillus casei cepa GG, Saccaromyces Boulardii, Bacterias productoras de ácido láctico (BAL) y Enterococcus SF68

- Es necesario, por tanto, definir mejor las especies, cepas, dosis, duración del tratamiento en cada una de las situaciones clínicas para hacer recomendaciones claras basadas en la evidencia.

\section{Los autores declaran no tener conflicto de interés.}

\section{REFERENCIAS}

1. Surawicz y Lawrence J, Brandt CM. probióticos y transplante de microbiota fecal. En: Sleisenger y Fordtran Enfermedades digestivas y hepáticas, La Ciudad Condal, España: Elsevier Masson, 2017, p 2339-2343, Disponible en: https://www.clinicalkey.es/\#!/content/book/3-s2.0B9788491132110001303?scrollTo $=\% 23 \mathrm{hl} 000024$ 9

2. Kashyap y Rebecca L. Morgan GAPAVWPC. AGA Technical Review on the Role of Probiotics in the Management of Gastrointestinal Disorders. Gatroenterology 2020 ;159(2):708-38. Disponible en:

https://www.clinicalkey.es/\#!/content/journal/1s2.0$\underline{\text { S0016508520347326? scrollTo }=\% 23 \mathrm{hl} 0000395 \%}$ $\underline{20}$

3. Garrote Antonieta, Bonet Ramon, Probióticos, Elsevier, Marzo 2017, Vol 31. Num 2, páginas 1316. Disponible en: https://www.elsevier.es/esrevista-farmacia-profesional-3-articuloprobioticos-X0213932417608720
4. Julio Plaza Diaz, Ruiz Ojeda Francisco, Gil Campos Mercedes, Gil Angel, Mechanisms of Actions of Probiotics, Advances in Nutrition, Volume 10, January 2019, pages S49-S66. Disponible

en: https://academic.oup.com/advances/article/10/su ppl 1/S49/5307225?login=true

5. Castañeda Guillot Carlos, Probióticos, puesta al dia, Scielo, Revista Cubana de Pediatría, 2018, Vol.90 no.2. Disponible en: http://scielo.sld.cu/scielo.php?script=sci arttext\& pid=S0034-75312018000200009

6. Susan Prescott, Christina West, Prebiotics and Probiotics for treatment of allergic disease, Uptodate, jan 22, 2020. Disponible en: https://www-uptodate-

com.binasss.idm.oclc.org/contents/prebioticsand-probiotics-for-treatment-of-allergicdisease? search=probioticos\&source=search res ult\&selectedTitle=4 138\&usage type=default\&di splay rank=4

7. Surawicz y Lawrence J. Brandt CM. probióticos y transplante de microbiota fecal. En: Sleisenger y Fordtran Enfermedades digestivas y hepáticas . la Ciudad Condal, España: Elsevier Masson; 2017. p. 2339-2343. Disponible en: https://www.clinicalkey.es/\#!/content/book/3-s2.0B9788491132110001303?scrollTo=\%23hl000024 $\underline{9}$

8. Food and Drug Administration (FDA). GRAS Notices. 2016 ago. Disponible en: https://www.cfsanappsexternal.fda.gov/scripts/fdc c/index.cfm?set=GRASNotices

9. Aneska Mariño Garcíal, Magdalys Núñez Velázquez, Jesús Barreto Peniéll. Microbiota, probióticos, prebióticos y simbióticos. IIServicio de Nutrición Clínica Hospital Hermanos Ameijeiras 2016.

10. Carlos Castañeda Guillot. Probióticos, puesta al día. Revista Cubana de Pediatría 2018; 90(2):286 $-298$

11. Segura Aurora, Que son los probióticos y porque los expertos de Harvard recomiendan su consumo, La Vanguardia, 10 febrero, 2021. Disponible en: https://www.lavanguardia.com/comer/tendencias/ 20210210/6230728/que-son-probioticosexpertos-harvard-recomiendan-consumo.html

12. Zanin Tatiana, Probióticos: que son, para que sirven y como tomarlos, Tua Saude dieta y nutrición, Abril 2021. Disponible en: https://www.tuasaude.com/es/que-son-losprobioticos/

13. American Gastroenterological Association (AGA). Choosing the right probiotics. Gastro.org. 2020. Disponible en: https://gastro.org/practiceguidance/gi-patient-center/topic/choosing-theright-probiotics $/$ ?hilite $=\% 27$ probiotics $\% 27$ 
14. Grace L. Su, Cynthia W. Ko, Premysl Bercik, Yngve Falck-Ytter, Shahnaz Sultan, Adam V. Weizman, and Rebecca L. Morgan. AGA Clinical practice guidelines on the role of probiotics in the management of gastrointestinal disorders. Gatroenterology 2020; 159:697-705

15. Berkeley N Limketkai, Anthony K Akobeng, Morris Gordon, Akinlolu Adedayo Adepoju, Probióticos para la inducción de la remisión en la enfermedad de Crohn, Cochrane Library 2020. https://doi.org/10.1002/14651858.CD006634.pub $\underline{3}$

16. Ruiz Abellan Maria, Probióticos para regular el estreñimiento, Instituto central lechera asturiana, 28 febrero 2020. Disponible en : https://www.39ytu.com/actualidad/ucamcapsa/probioticos-estrenimiento/

17. Gabriel Olveira, Inmaculada González-Molero. Actualización de probióticos, prebióticos y simbióticos en nutrición clínica. Endocrinología y Nutrición 2016; 63 (9): 482-494

18. Ballesteros-Pomar MD, González Arnaiz E. Papel de los prebióticos y los probióticos en la funcionalidad de la microbiota del paciente con nutrición enteral. Nutrición Hospitalaria 2018; 35(2):18-26

19. Castellano Cuesta Juan Antonio, Microbiota Intestinal y Hueso, Rev. Sociedad Val. Reuma. 2017, 7;2:1-4. Disponible en: https://dialnet.unirioja.es/descarga/articulo/64187 14.pdf

20. Wassermann AO. Síndrome Metabólico Definición. Epidemiología. Fepreva.org.

Disponible en: http://www.fepreva.org/curso/6to curso/material/ ut 1 2.pdf

21. Correa ML, Ojeda MS, Lo Presti MS. Consumo de prebióticos y probióticos en relación con marcadores de síndrome metabólico en estudiantes universitarios. Nutrición Clínica y Dietética Hospitalaria [Internet]. 05/2019;39(2):171-82. Disponible en: https://revista.nutricion.org/PDF/LOPRESTI.pdf 